\title{
COMMENT
}

METRICS Survey asks authors: is your most-cited work your best work? p.561
IMMUNOLOGY A history of autoimmune disease research and its pioneers $\mathbf{p . 5 6 4}$
MATHEMATICS John Napier book marks 400 years of logarithms p.566
CLIMATE 'Paris Protocol'

should boost trade in green technology $\mathbf{p . 5 6 7}$

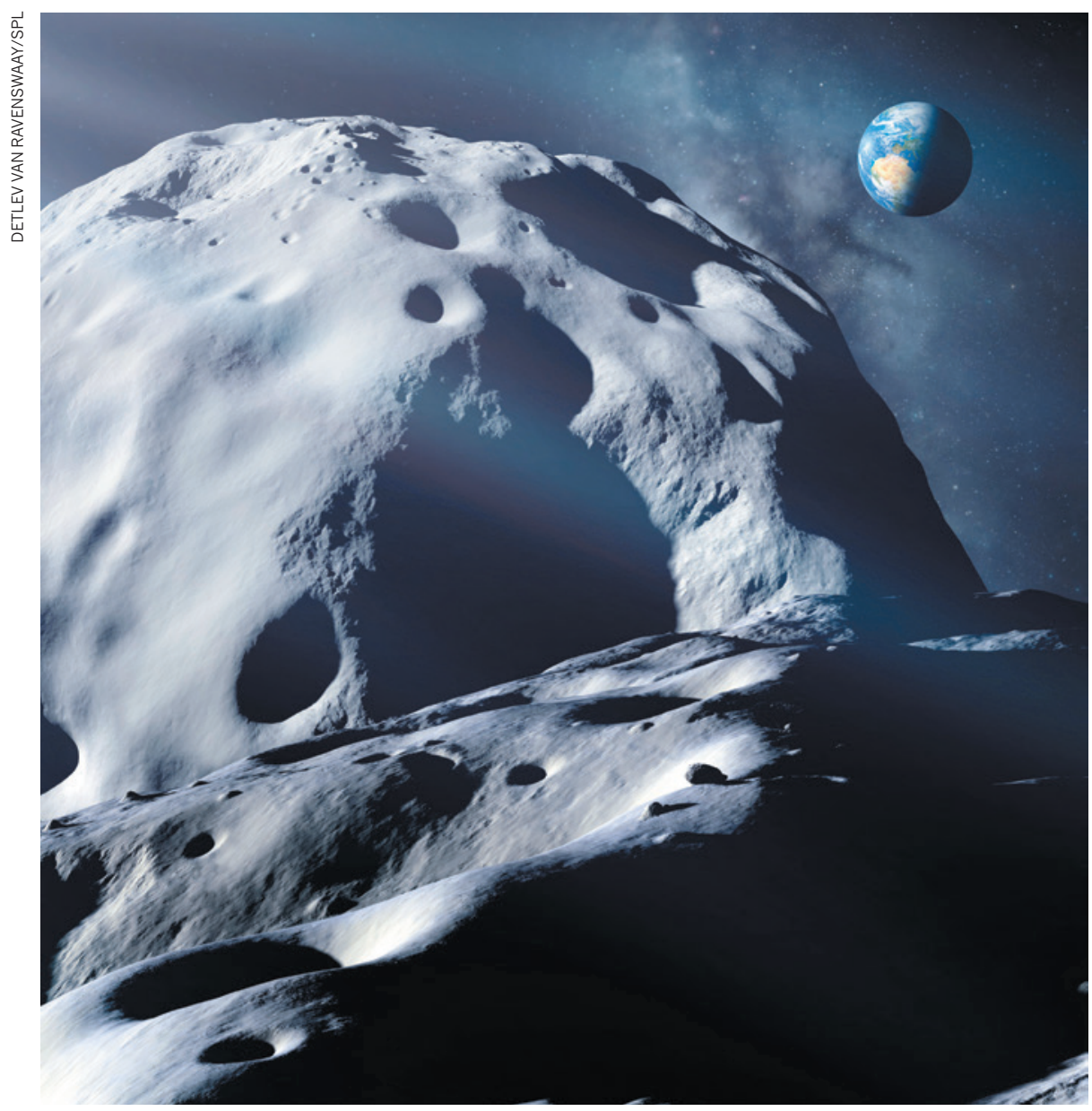

At least one roughly 10-metre-wide asteroid passes as close as the Moon each week (artist's impression).

\section{Find asteroids to get to Mars}

\section{Asteroid retrieval is a distraction, says Richard P. Binzel. Better steps to interplanetary travel abound.}

I nterplanetary flight is the next giant leap for humans in space. Yet consensus on even the smallest steps forward has proven elusive. In June, a US National Research Council report ${ }^{1}$ illuminated many options but offered no recommendations.
Return to the Moon? Head straight to Mars? Pluck a boulder off an asteroid and tug it to lunar orbit, just so that idle astronauts have somewhere to go and something to do? NASA must decide which path to follow before President Barack Obama's budget announcement in January 2015.

Some options are better than others. The cost and complexity of human space exploration demands that each element be measured by its value towards the ultimate goal: Mars.

But NASA's stated next priority will not contribute to that aim. Its Asteroid Redirect Mission $(\mathrm{ARM})^{2}$ is a multibillion-dollar stunt to retrieve part of an asteroid and bring it close to Earth where astronauts can reach it. It will require an ancillary spacecraft deploying either a huge capture bag or a Rube Goldberg contraption resembling a giant arcade-game claw. Neither technology is useful for getting humans to Mars.

There is a better way. Thousands of shipping-container-sized and larger asteroids pass almost as close as the Moon each year. Many, such as September's near-miss asteroid 2014 RC, come closer. We need to find them far enough in advance, and abundant opportunities for crewed missions will open up.

This gateway for human space exploration requires three things: a thorough asteroid survey to find thousands of nearby bodies suitable for astronauts to visit; extending flight duration and distance capability to everincreasing ranges out to Mars; and developing better robotic vehicles and tools to enable astronauts to explore an asteroid regardless of its size, shape or spin. The asteroid survey would also provide a prudent and overdue assessment of future impact hazards.

\section{PASSERS BY}

Asteroids orbit the Sun, most of them in the asteroid belt between Mars and Jupiter. Jupiter's gravity and other forces nudge a few onto paths that come within 40 million kilometres of Earth. The largest of these near-Earth asteroids (NEAs) is about 30 kilometres across, although most measure in metres. The smallest objects are most numerous and strike Earth frequently. Tonnes of their residual grains and pebbles rain down from space every day; a few metre-sized objects hit each year.

At about 20 metres across, the asteroid that lit the skies and shattered windows in Chelyabinsk, Russia, in 2013 demonstrated a threshold between merely delivering meteorites and posing a significant hazard. A Chelyabinsk-like airburst occurs somewhere on Earth on average every 50 years, usually over the oceans. A 10-kilometre-wide 
impactor such as the one that hit Earth at the end of the Cretaceous period is, thankfully, a one-in-hundred-million year event ${ }^{3}$.

But asteroids can be friends, not foes. For nearly four decades ${ }^{4}$, NEAs have been recognized as human spaceflight destinations that are more accessible than the lunar surface. Owing to their minuscule gravity fields, a rendezvous with an asteroid merely entails sidling up and flying alongside one, with no need for a specialized landing craft.

Asteroids whose orbits pass between Earth and Mars offer a range of milestone destinations for testing distance and duration capabilities of human spaceflight. The first missions might last weeks and not go far; later excursions could last months and venture successively farther into interplanetary space, proving that Mars is within our grasp.

A 2009 presidential task force ${ }^{5}$ recognized NEAs as a low-cost and achievable option along such a 'flexible path' to Mars. Obama set course gently by mentioning the word 'asteroid' and the year '2025' in a speech to a receptive crowd of space workers in April 2010. Although barely a murmur compared with President John F. Kennedy's exhortation in May 1961 to go to the Moon, NASA planners took Obama's words as a directive.

Reality soon set in: getting humans to any known near-Earth asteroid by the mid2020s was deemed beyond the range and budget of emerging flight systems. So the ARM scheme was hatched: instead of sending humans to an asteroid, let's bring a piece of one to within the anticipated reach of a 2025 crew. An uncrewed solar-electric propulsion vehicle would capture the booty and tug it back to a lunar orbit, where astronauts launched separately could explore it.

Mission accomplished? Not at all, in my view. Hardware and operations to capture, contain and redirect an asteroid are deadend elements with no value for long-duration crewed space travel ${ }^{1}$. Delivering a supply module to lunar space would be a more sensible way to demonstrate solar-electric propulsion and benefit astronaut safety.

"Why retrieve
an asteroid
when we can
wait for one to
come near us?"
Conveying to the public that reaching Mars requires patient and diligent progression in capabilities is the honest alternative to distracting them with a one-off costly stunt.

Equally specious in my opinion are arguments that ARM can deliver important new information on asteroid hazards or space resources. The ARM target would be scarcely one-quarter the size and onehundredth the mass of the Chelyabinsk body - too small to survive atmospheric passage. And the idea that we might benefit any time soon by extracting water or rocket fuel out of an asteroid is fantastical, owing to its cost and complexity.

\section{EXPLORATION GATEWAY}

NASA needs a more pragmatic plan. Rededication to Mars as the long-term goal is clearly necessary (independent of any decision regarding a return to the Moon). Near-Earth asteroids are the most accessible interplanetary stepping stones to Mars. Why retrieve an asteroid when we can wait for one to come near us?

There are plenty to choose from (see 'Mission requirements'). The roughly one thousand objects of tens of metres and larger ${ }^{6}$ currently known to pass within a few times the distance to the Moon are just the tip of the

\section{MISSION REQUIREMENTS}

A mission to a near-Earth asteroid would require less propulsion and a shorter mission duration than a human mission to Mars. Less than $1 \%$ of the most-accessible asteroids are currently known (yellow circles), but a dedicated survey (filling in the yellow-hatched region) would reveal abundant asteroid stepping-stone opportunities as a gateway for human interplanetary exploration.

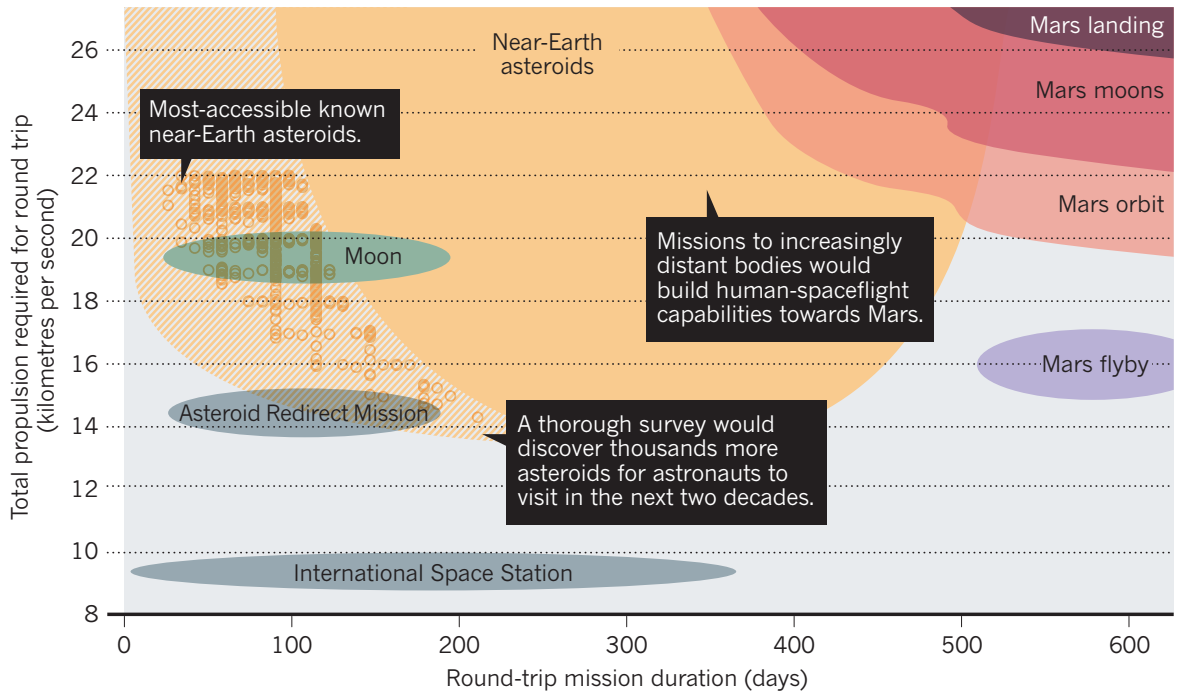

iceberg (see go.nature.com/9db89t). Nearly 10 million more such objects await discovery in orbits passing between Earth and Mars; one or more fly inside the Moon's orbit every week. So far, we have detected scarcely $0.1 \%$ of them because our current asteroid survey network is not up to the task.

The asteroids that are optimally located for exploration are also among those posing a potential impact hazard to Earth. 'Find them before they find us' has been the mantra of planetary scientists ${ }^{3,7}$ and national reports ${ }^{8,9}$ for decades. Yet neither the US Congress nor NASA has funded a large, dedicated telescopic survey. NASA spends only a minuscule fraction of its budget - \$US20 million per year - on searches using a haphazard array of adapted astronomical facilities.

Current surveys also fall short of US law. The George E. Brown, Jr. Near-Earth Object Survey Act of 2005 requires NASA to find $90 \%$ of hazardous asteroids 140 metres or larger by 2020, a goal that will not be met without significantly more investment. It is a grand challenge requiring a grand commitment from the space agency, the president and Congress: \$200 million per year. This budget line, comparable to NASA's New Frontiers programme that has sent uncrewed probes to the Solar System's planets, would allow the survey to be completed within a decade at a fraction of ARM's projected multibillion-dollar cost.

Once humans can reach one asteroid in its native orbit, the gateway is opened such that hundreds (if not thousands) more will be accessible, enabling a steady programme of exploration to be unrolled in the late 2020s and 2030s. Commercial development of space resources on select candidates could begin to be evaluated towards the middle of this century.

Robotic operated vehicles (ROVs) will need to be developed to explore asteroids, just as astronauts in orbit around or on the surface of Mars will need to command robotic workhorses. Most asteroids are irregularly shaped, boulder-strewn and tumble in their orbits. We have no reason to expect an accessible asteroid to have a slow and steady spin and safe, smooth surface hospitable to a space suit. ROVs such as those used for underwater exploration could be directed from the main crew module to carry out in situ investigations. With an array of arms or grappling devices to gain a toehold necessary for working in microgravity, and the capability to operate longer hours than possible for a spacewalk, exploration of these worlds could be achieved.

\section{GRAND CHALLENGE}

Over the next two months, Obama's 2015 budget will be shaped. NASA needs to make a clear choice about its priorities. It should abandon the ARM mission concept and 
make an asteroid survey its top priority to 5 provide a basis for future crewed missions.

NASA's directorates for human exploration, space technology, science and others must pool resources to address the agency's declared grand challenge. Congress and the White House should fund a new series of missions that embrace the resulting synergy of exploration, technology and science for the benefit of humanity.

Such a 'Grand Challenge Mission' programme should support human spaceflight and humankind's future. It should be modelled after, and with a budget similar to, the New Frontiers programme of planetary probes each costing less than $\$ 800$ million and selected through competition. Advance definition of objectives would drive development by industry and academia towards the best ideas and optimize long-term planning. Competitive selection of proposals ensures the most cost-effective return on taxpayer investment.

Three asteroid-related concepts should be explored: an asteroid survey to find a series of human destinations on the path to Mars while fulfilling the requirements of the 2005 survey act; a competition to test robotic asteroid-deflection methods on which civilization's survival could one day depend; and another to test robotic methods of extracting water or mining other valuable resources from asteroids that might contribute towards sustaining human spacefaring decades from now.

NASA needs to get back on track towards achieving humankind's next giant leap in space.

Richard P. Binzel is a joint professor of planetary science and aeronautics and astronautics at the Massachusetts Institute of Technology in Cambridge, Massachusetts, USA.

e-mail:rpb@mit.edu

1. National Research Council Pathways to Exploration: Rationales and Approaches for a U.S. Program of Human Space Exploration (National Academies Press, 2014).

2. NASA Asteroid Initiative Opportunities Forum Update on Asteroid Redirect Mission (NASA, 2014); available at go.nature.com/cu63ol.

3. Yeomans, D. K. Near-Earth Objects: Finding Them Before They Find Us (Princeton Univ. Press, 2013).

4. Shoemaker, E. M., Williams, J. G., Helin, E. F. \& Wolfe, R. F. in Asteroids (ed. Gehrels, T.) 253-282 (Univ. Arizona Press, 1979).

5. US Human Spaceflight Plans Committee Seeking A Human Spaceflight Program Worthy of a Great Nation (NASA, 2009).

6. Barbee, B. et al. in Proc. IAA Planet. Def. Conf. 2013 IAA-PDC13-04-13 (2013)

7. Chapman, C. R. \& Morrison, D. Nature 367, 33-40 (1994).

8. National Research Council Defending Planet Earth: Near-Earth Object Surveys and Hazard Mitigation Strategies (National Academies Press, 2010).

9. NASA Advisory Council Report of the Ad-Hoc Task Force on Planetary Defense (NASA, 2010).

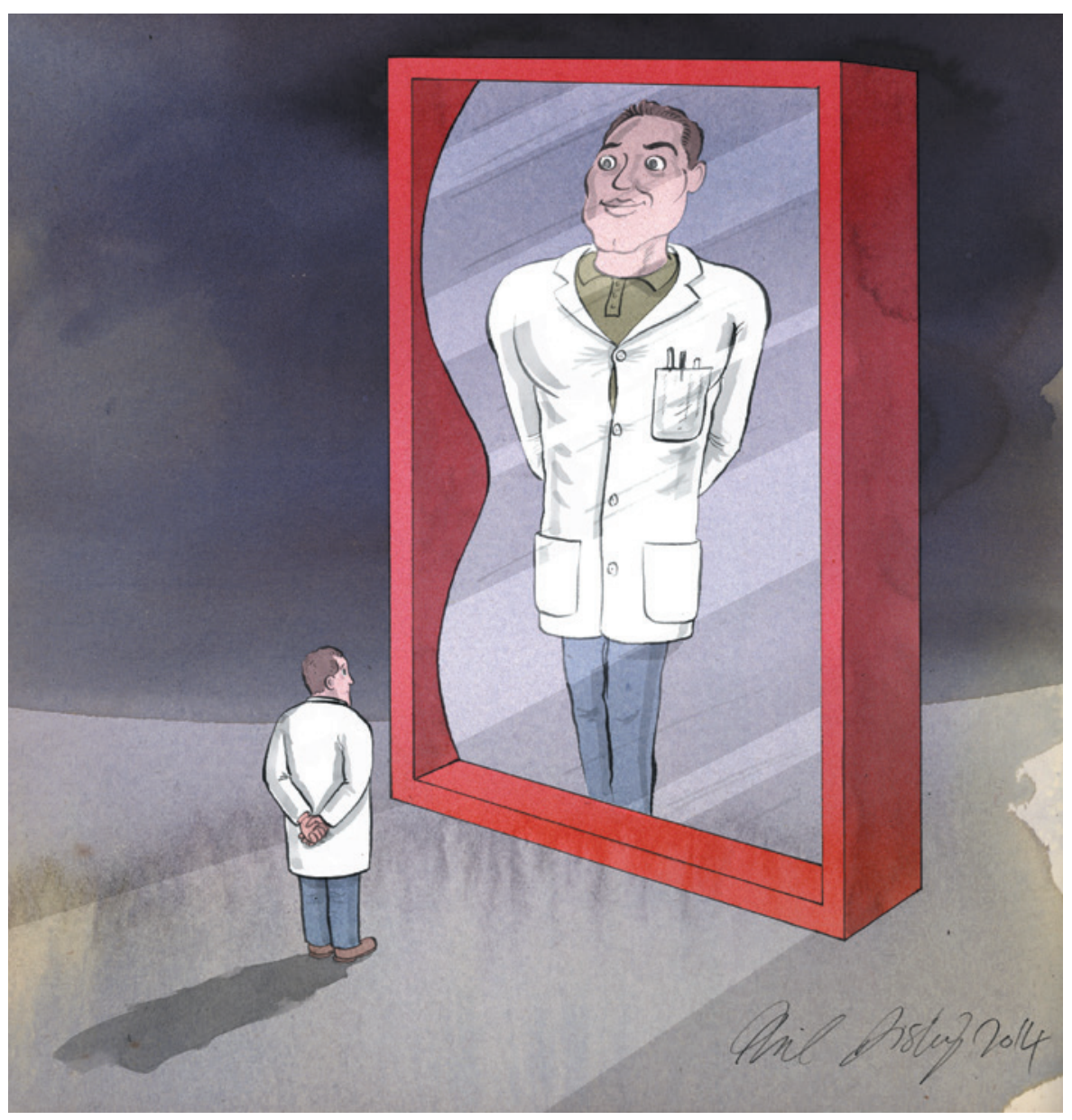

\section{Is your most cited work your best?}

\section{John P. A. Ioannidis and colleagues asked the most highly cited biomedical scientists to score their top-ten papers in six ways.}

A fter half a century of citation indices, several intriguing questions remain. Are the most highly cited papers the most important ones? Does science make progress mostly through evolution or through revolution? Are these two processes mutually exclusive or complementary, and which do high citations most reflect? Are surprising findings difficult to publish?

Highly cited papers are nodes in the network of the dissemination and discussion of scientific information. But citation counts alone cannot reveal why a paper is considered so important as to attract repeated mention by other researchers. To contribute to these debates, we surveyed the most-cited authors of biomedical research for their views on their own influential published work ${ }^{1-3}$

We got some intriguing feedback. The vast majority of this elite group felt that their most important paper was indeed one of their most-cited ones. Yet they described most of their chart-topping work as evolutionary, not revolutionary.

\section{BEST OF THE BEST}

We listed the 400 most-cited biomedical scientists in the period 1996-2011 $1^{4}$. We selected each author's ten most-cited papers (adjusted for publication year) published in 2005-08, and asked them to score the papers (on a scale of 0 to 100) in six dimensions.

We restricted the period to 2005-08, because the perception of the importance 